\section{$\$$ Research Square}

Preprints are preliminary reports that have not undergone peer review.

They should not be considered conclusive, used to inform clinical practice, or referenced by the media as validated information.

\title{
Microvirga Puerhi Sp. Nov., Isolated from Puerh Tea Garden Soil
}

\section{Zhi-Da Zi}

Beijing University of Chemical Technology

Wen Wen

Beijing University of Chemical Technology

Fu Ma

Beijing University of Chemical Technology

Wei Li

Beijing University of Chemical Technology

Zhao-Xuan Wang

Shijiazhuang People's Medical College

Jun Lu

Auckland University of Technology

Jie Lv ( $\boldsymbol{D}$ lvjie@mail.buct.edu.cn )

Beijing University of Chemical Technology https://orcid.org/0000-0003-4691-7562

\section{Research Article}

Keywords: Novel species, Microvirga, Polyphasic taxonomy, Genome, Puerh tea garden soil

Posted Date: January 3rd, 2022

DOI: https://doi.org/10.21203/rs.3.rs-1184891/v1

License: (c) (i) This work is licensed under a Creative Commons Attribution 4.0 International License.

Read Full License 


\section{Abstract}

Strain WGZ8 ${ }^{\top}$ was isolated from a soil sample of Puerh tea garden in Puer city, Southwest China. The isolate was rod-shaped, Gram-stain negative, aerobic, non-motile. Growth occurred within 0-3.0\% (w/v) $\mathrm{NaCl}$ (optimal concentration, $0-1.0 \%$ ), $\mathrm{pH} 5.0-11.0$ (optimal pH, 7.0) and $10-40^{\circ} \mathrm{C}$ (optimal temperature, $\left.28^{\circ} \mathrm{C}\right) .16 \mathrm{~S}$ rRNA gene sequences based phylogenetic and phylogenomic analysis revealed that WGZ8 ${ }^{\top}$ belonged to the genus Microvirga. Its major cellular fatty acids were $\mathrm{C}_{19: 0}$ cyclo $\omega 8 c, \mathrm{C}_{16: 0}, \mathrm{C}_{18: 1} \omega 7 c$ and/or $\mathrm{C}_{18: 1} \omega 6 \mathrm{c}$. The profile of polar lipids included phosphatidyldimethylethanolamine, phosphatidylmonomethylethanolamine, phosphatidylethanolamine, phosphatidylcholine, diphosphatidylglycerol and phosphatidyl-glycerol. The only respiratory quinone was detected as ubiquinone 10 (Q-10). The genome size of strain $\mathrm{WGZ8}^{\top}$ was $5.17 \mathrm{MB}$, and the content of DNA G+C was $61 \%$. Based on the results of digital DNA-DNA hybridization and phenotypic results, strain $\mathrm{WGZ8}^{\top}$ could be concluded as a novel species of the genus Microvirga, for which the name Microvirga puerhisp. nov. is

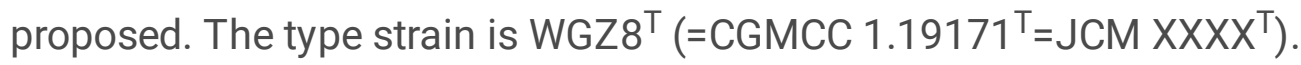

\section{Introduction}

The genus Microvirga, which was established by Kanso (Kanso et al. 2003), belongs to the family Methylobacteriaceae. According the LPSN database (https://lpsn.dsmz.de/genus/microvirga, Parte et al. 2020), 18 species with valid names were included in the genus Microvirga. Members of the genus Microvirga are aerobic, rod-shaped, oxidase negative and gram-negative. The predominant respiratory quinone is ubiquinone-10 (Q-10) and the G+C content of DNA is approximately 61.1 to $65.1 \mathrm{~mol} \%$ (Table S1). Members of genus Microvirga can adapt to a variety of ecological environments: air (Weon et al. 2010), geothermal waters (Kanso et al. 2003), hot spring sediment (Liu et al. 2021), forest soil (Zhang et al. 2019), and metal industry waste (Tapase et al. 2017). In this study, we propose a novel species of genus Microvirga, designated as WGZ8 ${ }^{\top}$ which was isolated from Puerh tea garden soil, using polyphasic taxonomy and genomic approaches.

\section{Materials And Methods}

\section{Isolation and culturing conditions}

Strain WGZ8 ${ }^{\top}$ was isolated from a soil sample from a Puerh tea garden, located in Pu'er City, Yunnan Province, China ( $22^{\circ} 42^{\prime} 35^{\prime \prime} \mathrm{N}, 100^{\circ} 56^{\prime} 10^{\prime \prime} \mathrm{E}$; altitude $\left.1130 \mathrm{~m}\right)$. Isolation and cultivation of microbials based on previously reported methods (Han et al., 2014). Strain $\mathrm{WGZ8}^{\top}$ was isolated on the oligotrophic Reasoner's $2 \mathrm{~A}$ agar (R2A) at $30{ }^{\circ} \mathrm{C}$ for 3 days and then stored in $15 \%(\mathrm{v} / \mathrm{v})$ glycerol suspension at $-80{ }^{\circ} \mathrm{C}$ for extended preservation.

Reference strains Microvirga subterranea DSM $14364^{\top}$ and'Microvirga rosea'MAH-2 were obtained from the Guangdong Microbiological Culture Collection Center (GDMCC). 


\section{Phylogenetic analysis}

16S rRNA gene of isolate WGZ8 ${ }^{\top}$ was amplified by PCR and sequenced as our previous report (Ma et al. 2021). EzBioCloud (Kim et al. 2011) (https://www.ezbiocloud.net/) was used to analyze the 16S rRNA gene sequence similarities with related strains. Maximum-parsimony (Walter et al. 1971), Neighborjoining (Saitou et al. 1987) and maximum-likelihood (Kishino et al. 1989) methods in MEGA 7 (Kumar et al. 2016) were used to construct phylogenetic trees. Enterovirga rhinocerotis YIM $100770^{\top}$ (KC992737) was used as an outgroup in the phylogenetic analysis. Other analysis methods were detailed in the footnotes of Fig. 1, Fig. S1 and S2.

\section{Genomic analysis}

Illumina Novaseq pe150 platform (Beijing Novogene Bioinformatics Technology Co., Ltd. Beijing China) was used to sequence the genome of strain $\mathrm{WGZ8}^{\top}$. The sequences were obtained and re-assembled as reported previously (Ma et al., 2021). As previously described (Galperin et al. 2015), the coding genes' functions were searched online using the COG server database (http://weizhonglab.ucsd.edu/webMGA/server/cog/). The NCBI Prokaryotic Genome Annotation Pipeline v5.0 was used to annotate, and OrthoFinder v2.2.6 was used to cluster the annotated genomes as described by Nguyen et al. (2015), and 1018 single copy orthologs were identified. Mafft software (Katoh et al. 2008) was used to align orthologs selected, then TrimAl was used to trim all the alignments. The best model automatically is GTR+F+R5. Maximum-likelihood tree was reconstructed using iQ-tree v1.6.12 as described by Castresana et al. (2000). ANI (average nucleotide identity, Konstantinidis et al. 2005) and dDDH (DNA-DNA hybridization, Auch et al. 2010) values between the strain $\mathrm{WGZ8}^{\top}$ and members of genus Microvirga was calculated using the web service www.ezbiocloud.net/tools/ani and http://ggdc.dsmz.de/distcalc2.php, respectively.

\section{Physiological properties analysis}

Colonies and cells of WGZ8 ${ }^{\top}$ were observed by eyes and the scanning electron microscopy (SU1510; Hitachi, Tokyo, Japan) after growing for 3 days at $28{ }^{\circ} \mathrm{C}$ on R2A agar. Nutrient agar (NA), Luria-Bertani agar (LB) and tryptone soya agar (TSA) were also used to test cell growth. Tests of anaerobic growth, Gram reaction, motility, oxidase, catalase, growth temperature, $\mathrm{pH}$ and $\mathrm{NaCl}$ tolerance were carried out using the methods reported previously (Ma et al., 2021). API ZYM, API 50CH and API 20NE kits were used to detect assimilation of acid production and enzyme activities, according to the manufacturers' instructions (BioMérieux SA, Marcy-l'Étoile, France). To investigate the hydrolysis of Tween 40 (w/v, $1 \%)$, Tween 80 (1\%), Tween 20 (1\%), CM-cellulose (0.5\%), casein (5 \% skimmed milk), and starch (0.2\%), R2A agar used as the basal medium, based on conventional methods (Smibert et al. 1994).

\section{Chemotaxonomic analysis}

Cells of reference strains and WGZ8 ${ }^{\top}$ were cultured and collected at $28{ }^{\circ} \mathrm{C}$ for 3 days on R2A. The collected cells were extracted, methylated and saponified as described before (Ma et al., 2021). 7890A GC 
system (Agilent, Santa Clara, USA) was used to analyze the fatty acid methyl-esters extracted from the above steps and the Sherlock Aerobic Bacterial Database (TSBA 6) was used to confirm compounds' identities. Ninhydrin, a-naphthol-sulfuric acid, molybdophosphoric acid and molybdenum blue were sprayed on TLC plates to identify polar lipids (Costa et al. 2011b; Minnikin et al. 1984). HPLC was used to extracted and analyzed respiratory quinones (Costa et al. 2011a).

\section{Results And Discussion}

\section{Phylogenetic and genomic analysis}

16S rRNA gene of strain WGZ8 ${ }^{\top}$ was sequenced (1451 bp). ' $M$. rosea' MAH-2shared the highest similarity (98.0 \%) with WGZ8 ${ }^{\top}$, followed by M. subterranea DSM $14364^{\top}(97.6 \%)$. The $16 \mathrm{~S}$ rRNA gene sequence similarity values were all lower than $98.65 \%$ of the recommended species cutoff (Kim et al. 2011). Fig. 1 (maximum-likelihood), Fig. S1 (neighbor-joining) and Fig. S2 (maximum-parsimony) revealed that strain WGZ8 $^{\top}$ should be a member of the genus Microvirga.

The genome size and DNA G+C content of strain $\mathrm{WGZ8}^{\top}$ were $5.17 \mathrm{MB}$ and $61.0 \%$, respectively (Table S1). One 16 S rRNA with $100 \%$ similarity to PCR results was detected from the genome. According to the analysis sequencing and GC depth, there was no contaminated sequence in the sample (Fig. S3). Table $\mathrm{S} 2$ showed the detail statistics of the genome analysis. The COG functional categories were listed in Table S3. The relationship between $\mathrm{WGZ8}^{\top}$ and related species was showed in phylogenomic tree (Fig. 2). Table $S 1$ showed the ANI and $\mathrm{dDDH}$ values between strain $\mathrm{WGZ}^{\top}$ and members of the genus Microvirga, ANI and dDDH values were all lower than the species delineation thresholds of $95-96 \%$ and $70 \%$, respectively. All these trees and analysis results support that $\mathrm{WGZ8}^{\top}$ should be a new species of the genus Microvirga.

\section{Physiological properties analysis}

WGZ8 ${ }^{\top}$ is Gram-negative and rod-shaped aerobic bacteria. It could grow well on R2A, TSA, NA and LB agar. Growth occurred at 0-3 \% (w/v) NaCl (optimal concentration, 0-1 \%), pH 5.0-11.0 (optimal pH, 7.0) and $10-40{ }^{\circ} \mathrm{C}$ (optimal temperature, $28^{\circ} \mathrm{C}$ ). After 3 days of incubation on R2A agar, colonies were smooth, translucent, dense and mass, wet, bright, convex, and circular with entire margins and milky white. Table 1 shows the phenotypic differences with reference strains. All the negative properties of strain $\mathrm{WGZ}^{\top}$ in commercial kits were listed in table S4.

\section{Chemotaxonomic characteristics}

The major fatty acids ( $>10 \%$ of total) of the strain $\mathrm{WGZ8}^{\top}$ were detected as $\mathrm{C}_{19: 0}$ cyclo $\omega 8 c(43.3 \%)$, $\mathrm{C}_{16: 0}(18.8 \%)$ and $\mathrm{C}_{18: 1} \omega 6 c$ and/or $\mathrm{C}_{18: 1} \omega 7 c(16.4 \%)$. The two reference strains fatty acid profile was similar to that of the $\mathrm{WGZ8}^{\top}$ (Table 2). The polar lipids of strain $\mathrm{WGZ8}^{\top}$ included phosphatidyldimethylethanolamine, phosphatidylmonomethylethanolamine, phosphatidylethanolamine, 
phosphatidylcholine, diphosphatidylglycerol and phosphatidylglycerol (Fig. S4). The respiratory quinone of strain WGZ8 ${ }^{\top}$ was the same to all members of the genus Microvirga, and was identified as Q-10 (100 $\%)$.

According to the chemotaxonomic, phenotypic and phylogenic data, $\mathrm{WGZ8}^{\top}$ can be considered as a new member of the genus Microvirga, for which the name Microvirga puerhi sp. nov. is proposed.

\section{Description of Microvirga puerhisp. nov.}

Microvirga puerhi (pu.erh'i. N.L. gen. n. puerhi of Pu-erh, referring to Pu-erh tea garden soil where the type strain was isolated).

Cells are rod-shaped, Gram-negative, aerobic, devoid of motility, approximately 0.4-0.8 $\mu \mathrm{m}$ wide and 1.5$2.5 \mu \mathrm{m}$ long. After growing in R2A agar at $28^{\circ} \mathrm{C}$ for 3 days, colonies are smooth, translucent, dense and mass, wet, bright, convex, and circular with entire margins, milky white., and 1-2 $\mathrm{mm}$ in diameter. Growth occurs within 0-3.0 \% (w/v) NaCl (optimal concentration, 0-1.0\%), pH 5.0-11.0 (optimal pH, 7.0) and 10-40 ${ }^{\circ} \mathrm{C}$ (optimal temperature, $28{ }^{\circ} \mathrm{C}$ ). It grows well on R2A, TSA, NA and LB agar. Positive for catalase and negative for oxidase; negative for hydrolysis of CM-cellulose, Tweens (20, 40 and 80$)$ and skim milk. Positive for starch and gelatin hydrolysis.

In API 20NE tests, it is positive for urease activity, D-glucose fermentation, assimilation of D-mannose, potassium gluconate, D-glucose and L-arabinose It is positive in API ZYM tests for naphthol-AS-BIphosphohydrolase, trypsin, esterase (C4), acid phosphatase, alkaline phosphatase and leucine arylamidase; weakly positive for valine arylamidase, esterase lipase (C8), $\beta$-glucosidase and $\beta$ galactosidase. In API $50 \mathrm{CH}$ tests, it is positive for acid production from L-arabinose, D-ribose, D-xylose, Dadonitol, aesculin ferric citrate, D-lyxose, D-fucose, L-fucose and potassium 5-ketogluconate; weakly positive for D-glucose. Negative properties in commercial kits are listed in table S4. The major cellular fatty acids are $\mathrm{C}_{19: 0}$ cyclo $\omega 8 c, \mathrm{C}_{16: 0}, \mathrm{C}_{18: 1} \omega 7 c$ and/or $\mathrm{C}_{18: 1} \omega 6 c$. The major polar lipids are phosphatidylethanolamine, phosphatidylcholine, diphosphatidylglycerol, phosphatidylglycerol, phosphatidyldimethylethanolamine and phosphatidylmonomethylethanolamine. Ubiquinone $10(\mathrm{Q}-10)$ is the only respiratory quinone.

The type strain, $\mathrm{WGZ8}^{\top}\left(=\mathrm{CGMCC1} 16488^{\top}=\mathrm{JCMXXXX^{ \top } )}\right.$ was isolated from a soil sample of Puerh tea garden in Puer city, Southwest of China. The genome size and DNA G+C content of the type strain is 5.17 $\mathrm{MB}$ and $61.0 \%$ respectively.

\section{Abbreviations}

ANI, average nucleotide identity; dDDH, digital DNA-DNA hybridization; COG, cluster of orthologous groups of proteins; RNA, ribonucleic acid.

\section{Declarations}


Acknowledgements

We thank Miantong Guo for her help with sample collection and strain isolation.

\section{Conflict of interest}

The authors declare that there is no conflict of interest.

\section{Ethical statement}

This study does not describe any experimental work related to human.

\section{References}

1. Auch AF, Von Jan M, Klenk H-P, Göker M (2010) Digital DNA-DNA hybridization for microbial species delineation by means of genome-to-genome sequence comparison. Stand Genomic Sci 2:117-134

2. Castresana J (2000) Selection of conserved blocks from multiple alignments for their use in phylogenetic analysis. Mol Biol Evol 17:540-552

3. Costa M, Albuquerque L, Nobre MF, Wait R (2011a) The extraction and identification of respiratory lipoquinones of prokaryotes and their use in taxonomy. Methods Microbiol 38:197-206

4. Costa M, Albuquerque L, Nobre MF, Wait R (2011b) The identification of polar lipids in prokaryotes. Method Microbiol 38:165-181

5. Galperin MY, Makarova KS, Wolf YI, Koonin EV (2015) Expanded microbial genome coverage and improved protein family annotation in the COG database. Nucleic Acids Res 43:261-269

6. Han L, Wu SJ, Qin CY, Zhu YH, Lu ZQ, Xie B, Lv J (2014) Hymenobacter qilianensis sp. nov., isolated from a subsurface sandstone sediment in the permafrost region of Qilian Mountains, China and emended description of the genus Hymenobacter. Antonie Van Leeuwenhoek 105:971-978

7. Huq, MA (2018) Microvirga rosea sp. nov.: a nanoparticle producing bacterium isolated from soil of rose garden. Arch Microbiol 200:1439-1445

8. Kanso S, Patel B (2003) Microvirga subterranea gen. nov., sp. nov., a moderate thermophile from a deep subsurface Australian thermal aquifer. Int J Syst Evol Microbiol 53:401-406

9. Katoh K, Toh H (2008) Recent developments in the MAFFT multiple sequence alignment program. Brief Bioinform 92:86-98

10. Kim OS, Cho YJ, Lee K, Yoon SH, Kim M, Na H, Park SC, Jeon YS, Lee JH, Yi H (2011) Introducing eztaxon-e: a prokaryotic 16s rRNA gene sequence database with phylotypes that represent uncultured species. Int J Syst Evol Microbiol 62:716-721

11. Kishino H, Hasegawa M (1989) Evaluation of the maximum likelihood estimate of the evolutionary tree topologies from DNA sequence data, and the branching order in hominoidea. J Mol Evol 29:170179 
12. Konstantinidis KT, Tiedje JM (2005) Genomic insights that advance the species definition for prokaryotes. Proc Natl Acad Sci U S A 102:2567-2572

13. Kumar S, Stecher G, Tamura K (2016) MEGA7: Molecular Evolutionary Genetics Analysis version 7.0 for bigger datasets. Mol Biol Evol 33:1870-1874

14. Liu ZT, Xian WD, Li MM (2021) Microvirga arsenatis sp. nov., an arsenate reduction bacterium isolated from Tibet hot spring sediments. Antonie Van Leeuwenhoek 113:1147-1153

15. Ma, F., Zi, ZD., Li, W (2021). Devosia sediminis sp. nov., isolated from subterranean sediment. Arch Microbiol 203, 4517-4523

16. Minnikin DE, O'Donnell AG, Goodfellow M, Alderson G, Athalye M, Schaal A, Parlett J.H (1984) An integrated procedure for the extraction of bacterial isoprenoid quinones and polar lipids. J Microbiol Methods 2:233-241

17. Nguyen LT, Schmidt HA, von Haeseler A, Minh BQ (2015) IQ-TREE: a fast and effective stochastic algorithm for estimating maximum-likelihood phylogenies. Mol Biol Evol 32:268-274

18. Parte, A.C., Sardà Carbasse, J., Meier-Kolthoff, J.P., Reimer, L.C. and Göker, M. (2020). List of Prokaryotic names with Standing in Nomenclature (LPSN) moves to the DSMZ. Int J Syst Evol Microbiol 70:5607-5612

19. Saitou N, Nei MC (1987) The neighbor-joining method: a new method for reconstructing phylogenetic trees. Mol Biol Evol 4:406-425

20. Smibert RM, Krieg NR (1994) Phenotypic characterization. In Methods for General and Molecular Bacteriology 2:607-654

21. Tapase SR, Mawlankar RB, Sundharam SS, Krishnamurthi S, Dastager SG, Kodam KM (2017) Microvirga indica sp. nov., an arsenite-oxidizing Alphaproteobacterium, isolated from metal industry waste soil. Int J Syst Evol Microbiol 67:3525-3531

22. Walter, M, Fitch (1971) Toward defining the course of evolution: minimum change for a specific tree topology. Syst Zool 20:406-416

23. Weon HY, Kwon SW, Son JA, Jo EH, Kim SJ, Kim YS, Kim BY, Ka JO (2010) Description of Microvirga aerophila sp. nov and Microvirga aerilata sp. nov., isolated from air, reclassification of Balneimonas flocculans Takeda et al. 2004 as Microvirga flocculans comb. nov. and emended description of the genus Microvirga. Int J Syst Evol Microbiol 60: 2596-2600

24. Zhang XJ, Zhang J, Yao Q (2019) Microvirga flavescens sp. nov., a novel bacterium isolated from forest soil and emended description of the genus Microvirga. Int J Syst Evol Microbiol 69:667-671

\section{Tables}

Table 1. Differential characteristics between strain $\mathrm{WGZ8}^{\top}$ and reference strains of species of the genus Microvirga. 


\begin{tabular}{|c|c|c|c|}
\hline Characteristic & 1 & 2 & 3 \\
\hline Colony colour & Cream-colored & light pink color & light pink color \\
\hline $\mathrm{pH}$ range for growth & $5-11$ & $5-7 *$ & $6-9 *$ \\
\hline \multicolumn{4}{|l|}{ Assimilation of (API 20NE): } \\
\hline Gelatin hydrolysis & - & - & + \\
\hline D-glucose & + & - & + \\
\hline L-arabinose & + & - & + \\
\hline D-mannose & + & - & + \\
\hline D-mannitol & - & + & - \\
\hline $\mathrm{N}$-acetylglucosamine & - & + & - \\
\hline Maltose & - & + & - \\
\hline Potassium gluconate & + & - & + \\
\hline \multicolumn{4}{|l|}{ Enzyme activities (API ZYM): } \\
\hline Esterase lipase (C8) & w & w & + \\
\hline$\beta$-Galactosidase & w & - & - \\
\hline$\beta$-Glucuronidase & - & - & w \\
\hline$\beta$-Glucosidase & w & w & - \\
\hline$N$-acetyl- $\beta$-glucosaminidase & - & w & w \\
\hline \multicolumn{4}{|l|}{$\begin{array}{l}\text { Acid production from } \\
\text { (API 50CH): }\end{array}$} \\
\hline Glycerol & - & w & - \\
\hline D-arabinose & - & w & - \\
\hline D-adonitol & + & - & + \\
\hline D-glucose & w & + & + \\
\hline D-fructose & - & - & w \\
\hline D-trehalose & - & + & - \\
\hline D-tagatose & - & w & + \\
\hline DNA G+C content $(\%)$ & 61.0 & $61.4 \neq$ & $65.1 \neq$ \\
\hline
\end{tabular}


Strains: 1, WGZ8' $;$; , 'Microvirga rosea' MAH-2; 3, Microvirga subterranea DSM 14364' ${ }^{\top}$. Symbol: +, positive; -, negative; w, weakly positive. Data were taken from this study unless otherwise indicated. All strains were positive for the following characteristics: D-glucose fermentation, alkaline phosphatase, esterase (C4), leucine arylamidase, valine arylamidase, trypsin, acid phosphatase, naphthol-AS-BIphosphohydrolase, L-arabinose, D-ribose, D-xylose, aesculin ferric citrate, D-lyxose, D-fucose, L-fucose, potassium 5-ketogluconate.

*Results from the previously reports (Huq, MA 2018; Sungwan et al. 2003)

$\ddagger$ Data from the genome sequences.

Table 2. Cellular fatty acid profiles of strain $\mathrm{WGZB}^{\top}$ and reference strains Strains: $1, \mathrm{WGZB}^{\top}$; 2 , 'Microvirga rosea' MAH-2; 3 , Microvirga subterranea DSM $14364^{\top}$. All data were from this study. Values are percentages of the total fatty acids; fatty acids amounting to $<1 \%$ of the total fatty acids are not listed; TR, trace (<1\%); ND, not detected.

\begin{tabular}{|llll|}
\hline Fatty acid & 1 & 2 & 3 \\
\hline Straight-chain & & & \\
\hline $\mathrm{C}_{16: 0}$ & 18.8 & 5.2 & 8.2 \\
\hline $\mathrm{C}_{17: 0}$ & 1.9 & $\mathrm{TR}$ & 6.4 \\
\hline $\mathrm{C}_{18: 0}$ & 4.4 & $\mathrm{TR}$ & 3.9 \\
\hline Cyclo & & & \\
\hline $15: 1 \omega 8 c$ & $\mathrm{ND}$ & 1.3 & $\mathrm{TR}$ \\
\hline $\mathrm{C}_{17: 0}$ cyclo & 4.2 & $\mathrm{TR}$ & $\mathrm{ND}$ \\
\hline $\mathrm{C}_{17: 1} \omega 6 \mathrm{c}$ & $\mathrm{ND}$ & $\mathrm{ND}$ & 1.5 \\
\hline $\mathrm{C}_{18: 1} \omega 7 \mathrm{c} 11$-methyl & 3.8 & $\mathrm{TR}$ & 1.4 \\
\hline $\mathrm{C}_{19: 0}$ cyclo $\omega 8 \mathrm{c}$ & 43.3 & 17.8 & 29.4 \\
\hline $\mathrm{C}_{20: 2} \omega 6,9 c$ & 2.5 & $\mathrm{TR}$ & $\mathrm{TR}$ \\
\hline Summed features* & & & \\
\hline 2 & 3.3 & 3.2 & 3.1 \\
\hline 3 & 1.3 & 2.8 & 1.2 \\
\hline 8 & 16.4 & 66.6 & 41.7 \\
\hline
\end{tabular}

Page $9 / 10$ 
*Fatty acids that could not be separated by GC using the MIDI system. summed feature 2, $\mathrm{C}_{12: 0}$ aldehyde, $\mathrm{C}_{14: 0} 3 \mathrm{OH}$ and/or $\mathrm{C}_{16: 1}$ iso $\mathrm{l}$; summed feature $3, \mathrm{C}_{16: 1} \omega 7 \mathrm{c}$ and/or $\mathrm{C}_{16: 1} \omega 6 \mathrm{c}$, summed feature 8, $\mathrm{C}_{18: 1} \omega 7 c$ and/or $\mathrm{C}_{18: 1} \omega 6 c$.

\section{Figures}

\section{Figure 1}

Maximum-likelihood phylogenetic tree based on 16S rRNA gene sequences showing the taxonomic position of strain $\mathrm{WGZB}^{\top}$ in the genus Microvirga. Numbers at nodes indicate bootstrap percentages (based on 1,000 replications); values $>50 \%$ are shown. Bar, 0.01 substitutions per nucleotide position. Enterovirga rhinocerotis YIM 100770 ${ }^{\top}$ (KC992737) was used as an outgroup.

\section{Figure 2}

Phylogenemic relationship of strain $\mathrm{WGZ8}^{\top}$ and closely related strains in the genus Microvirga. The phylogenemic tree of strain WGZ8 ${ }^{\top}$ and related type strains of the genus Microvirga based on genes with homology and 1018 single copies in 22 genomes sequences. Bar, 0.1 nucleotide substitution rate (Knuc) units. The relationships were inferred using the maximum-likelihood algorithm using iQ-tree v1.6.12. Enterovirga rhinocerotis YIM $100770^{\top}$ was used as an outgroup.

\section{Supplementary Files}

This is a list of supplementary files associated with this preprint. Click to download.

- WGZ8SuppplementarymaterialAOMI.docx 